\title{
Borderline Brenner tumor with a concomitant mucinous tumor of the ovary: a case report
}

\author{
Hülya Ayık Aydın, Zeynep Bayramoğlu², Armağan Aydın, Hadice Elif Pestereli², Tayup Şimşek ${ }^{1}$ \\ ${ }^{I}$ Department of Obstetrics and Gynecology, Division of Gynecologic Oncology, Akdeniz University School of Medicine, Antalya, \\ Turkey \\ ${ }^{2}$ Department of Pathology, Akdeniz University School of Medicine, Antalya, Turkey \\ ${ }^{3}$ Department of Medical Oncology, Antalya Training and Research Hospital, Antalya, Turkey
}

\begin{abstract}
Brenner tumors (BTs) are rare ovarian tumors which are a part of the epithelial stromal tumor group of ovarian neoplasms. Most of the BTs are benign and usually asymptomatic. BTs stand for the 1.4-2.5\% of ovarian tumors. Of the BTs, not more than $2 \%$ exhibit characteristics of borderline (or malignant) BTs. Borderline BTs are usually bigger than benign BTs and individuals typically represent with symptoms related with a unilateral ovarian mass. BTs are often related with mucinous cystadenomas or seldom cystadenocarcinomas. We present a case of borderline BT associated with a concomitant mucinous ovarian tumor.
\end{abstract}

Eur Res J 2018;4(2):122-125

Keywords: Brenner tumor, borderline Brenner tumor, ovarian tumor

\section{Introduction}

Brenner tumors (BTs) are defined by Fritz Brenner as oophoroma folliculare ovarii in 1907, and comprise $5 \%$ of benign tumors of the ovary [1]. They are comprised of layers of urothelium-like epithelial cells, that are encircled by condensed fibrous stroma and are thought to rise from superficial epithelium of the ovary that suffers transitional metaplasia. BTs comprise nearly $1.4-2.5 \%$ of tumors of the ovary [2]. World Health Organization categorized BTs into 3 groups: malignant, borderline and benign. The benign ones are the most frequent, representing about $95 \%$, the borderline represent about $5 \%$, and the malignant ones less than $1 \%$ [3]. Borderline BTs are epithelial tumors that appear as cellular islands or irregular epithelial masses that predominate in the cystic areas, differentiated by a dense conjunctive tissue at the periphery. BTs are more frequently related with cystadenomas or infrequently cystadenocarcinomas that are thought to be a kind of BT's metaplastic variation [4]. We present a case of borderline BT associated with a concomitant mucinous ovarian tumor. 


\section{Case Presentation}

A 54-year-old postmenopausal G2P2 female patient applied to our clinic with the complaints of abdominal distension, and inguinal pain which she noted two months ago. Medical history of the patient was unremarkable. Pelvic computed tomography demonstrated a multicystic lesion measuring $26 \times 23 \times 12 \mathrm{~cm}$ with a solid component, freely floating fluid around the mass and in the pelvic area (ascites?), millimetric soft tissue densities in the mesenteric adipose tissue at the left side of the mass, and blurred/thickened omentum lateral to the mass (peritoneal carcinomatosis?) (Figure 1). Preoperative tumor marker CA-125 was measured as $33 \mathrm{kU} / \mathrm{L}$.

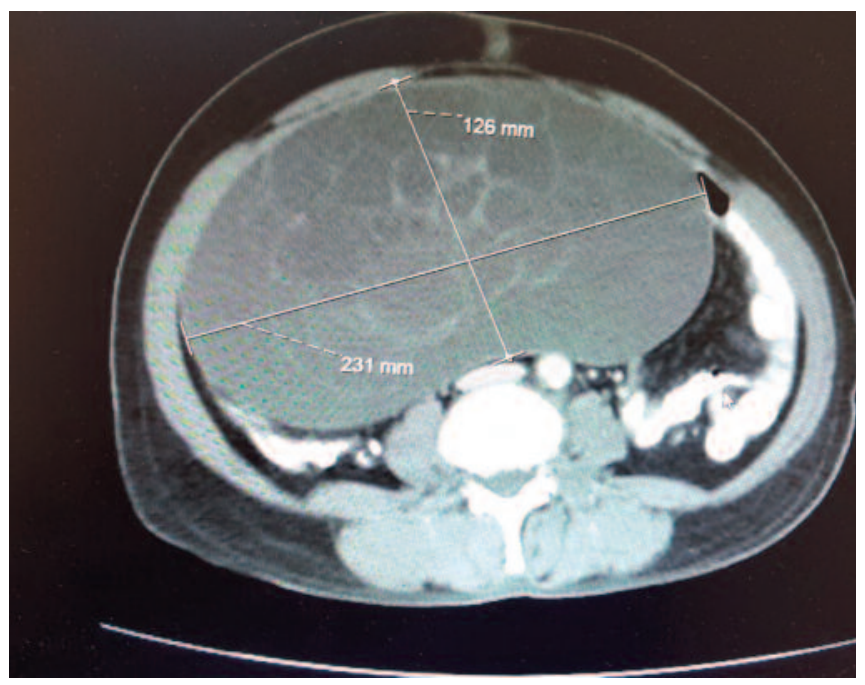

Figure 1. Pelvic computed tomography showing a multicystic lesion measuring $26 \times 23 \times 12 \mathrm{~cm}$ with a solid component

Midline laparotomy was performed. Abdominal exploration revealed a $30 \mathrm{~cm}$-bulky tumor originating from the left ovary (Figure 2). Contralateral ovary was apparently normal. The result of frozen section performed during surgery was interpreted as a "malign" lesion. The patient underwent total abdominal hysterectomy, bilateral salpingooophorectomy, bilateral pelvic paraaortic lymphadenectomy, infracolic omentectomy, appendectomy, and multiple peritoneal biopsies were also performed.

Postoperative pathology was interpreted as "borderline BT originating from the left ovary and concomitant mucinous ovarian tumor". Histopathological analysis of all other samples were reported as normal. On immunohistochemical staining, p-63-positive, while WT-1 negative nuclei were observed (Figure 3).

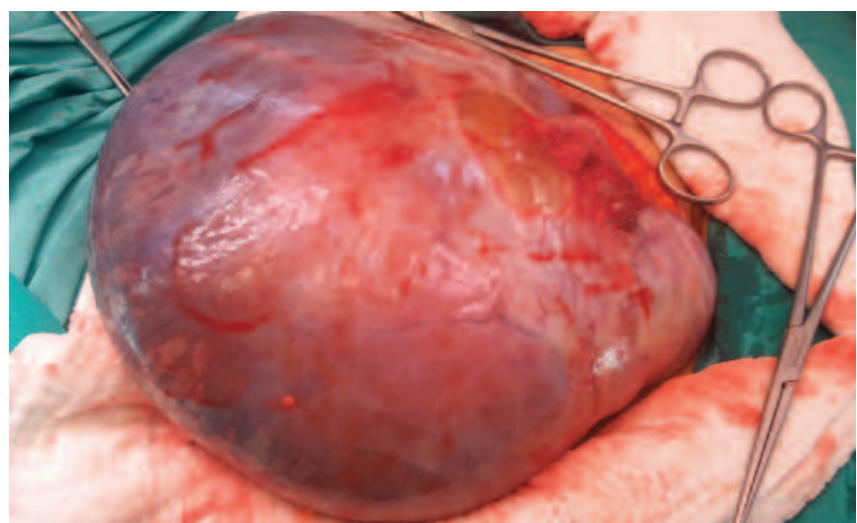

Figure 2. A $30 \mathrm{~cm}$-bulky tumor originating from the left ovary.

The patient who did not experience any complication during postoperative period was discharged. Follow-up of this stage 1A patient was planned. From January 2017, up to now she hasn't experience any disease recurrence.

\section{Discussion}

Benign BTs are seen during the 4th or 5th decade, while borderline and malignant BTs appear approximately 10 years later. Individuals commonly pose with symptoms related with an ovarian mass at one side. Borderline BTs are characteristically bigger than benign BTs, with an average size of 16 to $20 \mathrm{~cm}$. Abdominal exploration of the present case revealed a $30 \mathrm{~cm}$-bulky tumor originating from the left ovary. Contralateral ovary was apparently normal.

p-63 immunoexpression has been confirmed to be a valuable sign in normal uroepithelium and upper urinary tract. Earlier morphological reports have revealed that BTs display urothelial differentiation [5]. Liao et al. [6] revealed that $\mathrm{p}-63$ is shown in all benign and borderline BTs, and also revealed that most malignant BTs had deficiency of p-63 immunoexpression, implies that p-63 may take role in ovarian BTs pathogenesis. In the present report, the patient showed p-63-positivity.

Typical definition of an ovarian BT by computed tomography and magnetic resonance imaging is broad and vague calcification in a solid element in a multilocular cystic lesion [7]. On computed tomography, the benign component contains intense calcifications; whereas on magnetic resonance imaging, benign component shows very low intensity on T2-weighted images, and malignant component 


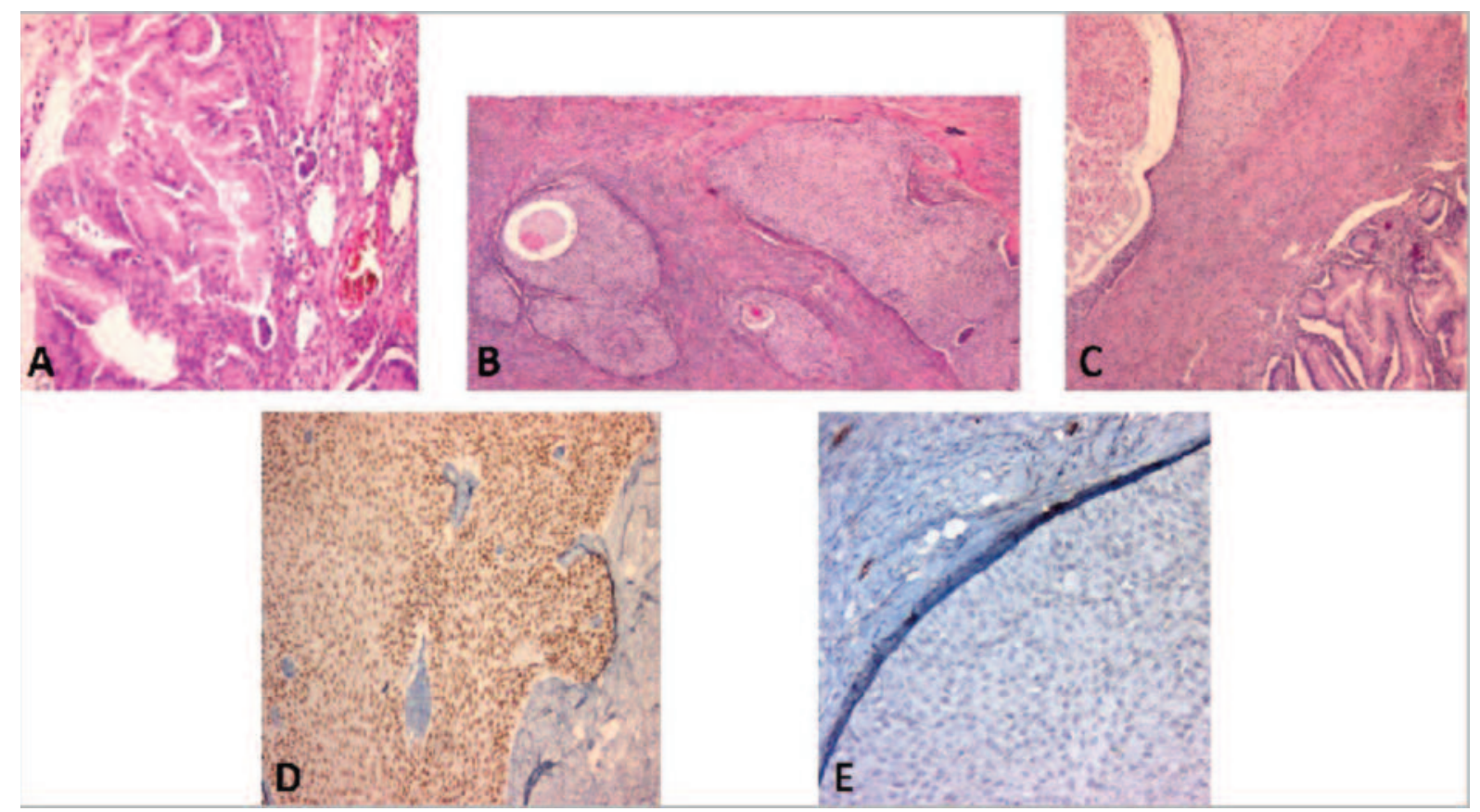

Figure 3. A. Mucinous boderline tumor with microinvasive carcinoma (H\&E stain 10×). B. Atypical proliferative (borderline) Brenner tumor. Large nest of proliferating transitional-type epithelium in center of field with extensive stratification (H\&E stain $5 \times$ ). C. Mucinous boderline tumor and Brenner borderline tumor. (H\&E stain $5 \times$ ). D. Atypical proliferative (borderline) Brenner tumor. Immunohistochemical stain for p63 is positive $(10 \times)$. E. Atypical proliferative (borderline) Brenner tumor. Immunohistochemical stain for WT-1 is negative $(20 \times)$.

shows high intensity. In the present report, pelvic computed tomography demonstrated a multicystic lesion with a solid component, freely floating fluid around the mass and in the pelvic area.

The borderline BT of the ovary continually had a pleasant prognosis and show a benign clinical progression after oophorectomy. The greater part of BTs are benign; thus, timely and precise detection of a malignant tumor would confirm that the individual obtains the exact surgery required [8].

Brenner tumors are frequently related with cystadenomas or infrequently with cystadenocarcinomas [4]. It is noteworthy that nearly $1 / 4$ of mucinous ovarian tumors, a small BT element is detected [9]. The co-existence of germ-cell tumors such as mature cystic teratoma, detected in our report as well, though infrequent is deliberated by individual authors as sign of a germ cell origin of the BTs $[4,9]$. Most BTs are benign. Strict measures similarly present for the categorization of borderline BTs. A new classification of borderline BTs is suggested: grade 1 (borderline, not otherwise specified) and grade 2-3 (borderline with intra-epithelial carcinoma) [9]. Most of the borderline BTs are detected in the first stage and have a favorable progression after the surgical treatment, with a survival rate of five years in about $99 \%$ of cases, and of 10 years in about $97 \%$ of cases [10].

\section{Conclusion}

We describe a case of borderline BT associated with a concomitant mucinous ovarian tumor. The positive diagnosis was done after surgery through the classical histopathological evaluation associated with immunohistochemical examinations. The progression after surgery was a favorable one, there were not detected any abdominal relapses during the ultrasound examination.

\section{Informed consent}

Written informed consent was obtained from the patient for the publication of this case report.

\section{Conflict of interest}

The authors declared that there are no potential conflicts of interest with respect to the research, authorship, and/or publication of this article.

\section{Acknowledgements}

This paper was presented as poster at the 20th Biennial Meeting of the European Society of Gynaecological Oncology (ESGO 2017), November 4-7, 2017, Vienna, Austria. 


\section{References}

[1] Brenner F. Das Oophoroma Folliculare. Frankfurt Z Pathol 1907;1:150-71.

[2] Roma AA, Masand RP. Ovarian Brenner tumors and Walthard nests: a histologic and immunohistochemical study. Hum Pathol 2014;45:2417-22.

[3] Lee KR, Tavassoli FA, Prat J. Surface epithelial-stromal tumors. In: Tavassoli FA, Devilee P (eds). Pathology and genetics of tumors of the breast and female genital organs. World Health Organization (WHO) Classification of Tumors, International Agency for Research on Cancer (IARC) Press, Lyon, 2003, pp. 140-3.

[4] Nomura K, Aizawa S. A histogenetic consideration of ovarian mucinous tumors based on an analysis of lesions associated with teratomas or Brenner tumors. Pathol Intern 1997;47:862-5.

[5] Roth LM. The Brenner tumor and the Walthard cell nest: an electron microscopic study. Lab Invest 1974;31;15-23.

[6] Liao XY, Xue WC, Shen DH, Ngan HY, Siu MK, Cheung AN. p63 expression in ovarian tumors: a marker for Brenner tumors but not transitional cell carcinomas. Histopathology 2007;51:477-83.

[7] Murat A, Ozdemir H, Yildirim H, Arslan A, Ersoy F, Ozercan R. Bilateral malignant Brenner tumor of the ovary. J Womens Imaging 2005;7:126-30.

[8] Hermanns B, Faridi A, Rath W, Fuzesi L, Schroder W. Differential diagnosis, prognostic factors, and clinical treatment of proliferative Brenner tumor of the ovary. Ultrastruct Pathol 2000;24:191-6.

[9] Scully RE, Young RH, Clement PB. Tumors of the ovary, maldeveloped gonads, fallopian tube and broad ligaments. Armed Forces Institute of Pathology, Washington, pp 153-63, 1998.

[10] Daraï E, Fauvet R, Uzan C, Gouy S, Duvillard P, Morice P. Fertility and borderline ovarian tumor: a systematic review of conservative management, risk of relapse and alternative options. Hum Reprod Update 2013;19:151-66. 